\title{
Computer Simulation of Hole Distribution in Polymeric Materials
}

\author{
Hélder M. C. Barbosa ${ }^{1, a}$ and Marta M. D. Ramos ${ }^{1, b}$ \\ ${ }^{1}$ Departamento de Física, Universidade do Minho, Campus de Gualtar, 4710-057 Braga, Portugal \\ ahelder@fisica.uminho.pt, ${ }^{\mathrm{b} m a r t a @ f i s i c a . u m i n h o . p t}$
}

Keywords: Mesoscopic modelling, PPV, polymer morphology, space charge distribution

\begin{abstract}
Polymers have been known for their flexibility and easy processing into coatings and films, which made them suitable to be applied in a variety of areas and in particular the growing area of organic electronics. The electronic properties of semiconducting polymers made them a serious rival in areas where until now inorganic materials were the most used, such as light emitting diodes or solar cells. Typical polymers can be seen as a network of molecular strands of varied lengths and orientations, with a random distribution of physical and chemical defects which makes them an anisotropic material. To further increase their performance, a better understanding of all aspects related to charge transport and space charge distribution in polymeric materials is required. The process associated with charge transport depends on the properties of the polymer molecules as well as connectivity and texture, and so we adopt a mesoscopic approach to build polymer structures. Changing the potential barrier for charge injection we can introduce holes in the polymer network and, by using a generalised Monte-Carlo method, we can simulate the transport of the injected charge through the polymer layer caused by imposing a voltage between two planar electrodes. Our results show that the way that holes distribute within polymer layer and charge localization in these materials is quite different from the inorganic ones.
\end{abstract}

\section{Introduction}

A semiconducting polymer network has a "spaghetti" structure where each chain is made of conjugated segments with different lengths connected by kinks and twists. Depending on the processing conditions, the polymer sample can have domains with a particular morphology (crystalline or amorphous) characterized by different chain orientations [1]. When charges are injected into this type of material, they distribute themselves in a particular manner in the polymer network. This charge distribution can influence the efficiency of electronic devices based on polymers. The mobility of charge carriers in these polymer layers depends, among other factors, on the properties of the polymer molecules, the existence of defects and the arrangement of these chains in the polymer network. Variations in molecular weight distribution, free volume, chemical irregularities, conformational defects or cross-links between chains constitute some defects that turn out to be an obstacle for charge transport [2]. But even in defect-free polymer samples, charge transport is limited by the molecular properties (e.g. ionization potential, electron affinity and intramolecular charge mobility) of the polymer strands and the sample morphology. It is known that molecular properties vary with the length of polymer segments [3]. Therefore, even defect-free samples containing segments with different lengths will have a distribution of localized sites where, depending on the local electric field, carriers can be trapped (the local electric field in a particular point of the polymer network corresponds to the sum of the electric field due to space charge distribution, the applied electric field and the field due to electrode polarization). In this paper we use a generalized Monte Carlo method to simulate the transport of charge carriers in a defect-free poly(p-phenylene vinylene) (PPV) network with three different chain orientations relative to the electrodes (parallel, perpendicular and random) to gain insight into the influence of the morphology and molecular properties of PPV chains in the distribution of positive charges in the network.

\section{Device Model and Simulation Method}

We constructed polymer networks by considering that the polymer chains are made of stiff-chain segments with different lengths. In our polymer layer, with $100 \mathrm{~nm}$ thickness, the number of 
monomers that exist in each chain segment obeys a Gaussian distribution with an average segment length of 5 monomers [4]. In our simulations we use polymer layers in which the polymer segments are either parallel, perpendicular or randomly oriented relative to the electrode. In this way we simulate the possible chain orientations resulting from different processing conditions [3] . Then we simulated charge injection and transport among these polymer structures using an improved version of our previously described model [4-6]. In the current version of the model we have explicitly modelled the electrodes: the charge injection now depends on the characteristics of the potential barrier at electrode/polymer interfaces. In our model the potential barrier height is dictated by the work function of the electrodes used, the properties of the conducting polymer molecules, the space charge distribution in the polymer network and the electrode polarization. We consider that the electrodes used are good conductors with perfect texture and without any impurities. Therefore, the only physical parameter that we have taken into account was their work function. One the other hand, the presence of a charge in the polymer layer will polarize the electrodes and as a result an image charge is created on both electrodes. All these aspects, besides the electric potential due to the applied bias, have to be considered in the calculation of the potential barrier height before a charge is injected. Since through out the polymer layer the length of the polymer strands are not the same, and the properties of each polymer strand depend on its length (different conjugated segment length means different ionization potential and electron affinity) [4], we will have near the electrode/polymer interfaces a cascade of potential barriers. Since our objective in this work is to understand the distribution of holes in the polymer network, until the internal electric field allows electron injection, we imposed a work function to the cathode high enough so no electron is injected due to the applied bias. We have changed the applied electric field and the polymer morphology to gain insight in how these factors will affect hole distribution in the polymer layer. This kind of detailed knowledge can only be achieved by mesoscopic computational methods.

\section{Results and Discussion}

As we increase the applied electric field, the number of holes present in the polymer layer increases for all morphologies, as we can see in Fig.1. That is not surprising since more holes will be able to tunnel through the potential barrier at anode/polymer interface.

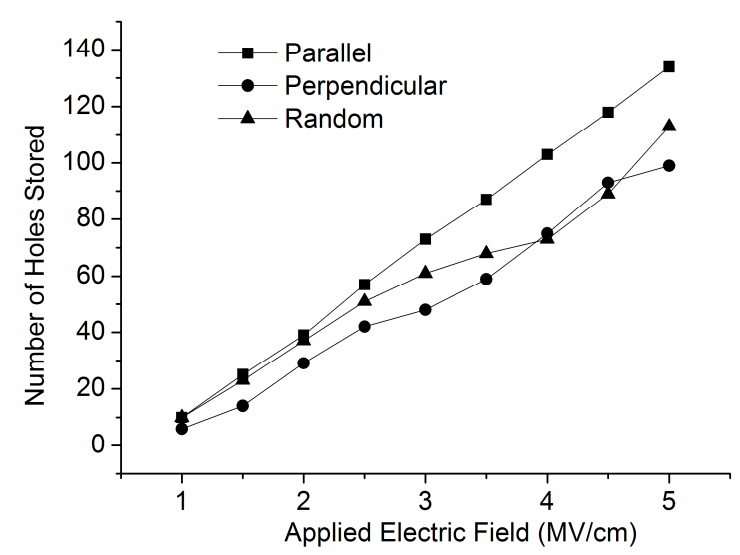

Fig. 1 - Number of holes stored in the polymer layer as a function of the applied electric field for three different chain orientations relative to the electrodes: parallel (squares), perpendicular (circles), random orientation (triangles). The lines are just a guide to the eye.

But for the same strength of the applied electric field, the total number of holes stored is not the same for polymer layers with different chain orientations. The reason for this behaviour is related to the dependence of intra-molecular mobility (mobility along a polymer strand) with the polymer chain orientation relative to the electrodes and the applied electric field. When the polymer chains are parallel to the electrodes, intra-molecular charge mobility will not contribute significantly for 
charge percolation towards the target electrode opposite to the injection electrode. In this case the applied electric field is normal to the chain axis and, therefore, only changes in the local electric field, due to space charge distribution, can allow a hole to move along a chain. The opposite occurs for the polymer chains with random and perpendicular orientation relative to the electrodes, since the applied electric field will contribute to charge mobility along a polymer strand and so intramolecular mobility will influence charge percolation. In polymer networks with these morphologies hole percolation is easier and, consequently, the number of holes stored inside the polymer layer is smaller. The statement above is not true for all strengths of the applied electric field, since when the strength of the electric field along the polymer chain is lower than a certain value, no intramolecular charge mobility occurs and this transport process does not affect hole percolation.As a result, for an applied electric field of $1 \mathrm{MV} / \mathrm{cm}$ the total number of holes present in the polymer network is similar for the three morphologies considered in this work. This happens because the local electric field along the polymer strands is smaller than the minimum threshold needed for the mobility of holes along a polymer strand $(1.951 \mathrm{MV} / \mathrm{cm})$ [5]. Therefore, the hole mobility through the polymer layer is realized by hopping between neighbouring chains (intra-molecular charge mobility), a process which is independent of the orientation of the polymer strands. Since the polymer films have approximately the same density, regardless of their molecular orientation, and thus approximately the same number of chains, we can conclude that hole percolation in this case is due essentially to inter-molecular mobility. Therefore, potential barriers between hole hopping sites will be the dominant effect in charge distribution in the polymer network for low applied electric fields and hole distribution in this case will not be dependent of polymer chain orientation. On the other hand, when the applied electric field is high enough, the morphology of the sample will play an important role for hole distribution. For all polymer films with different morphologies, holes are distributed inside the polymer network in such a way that a gradient in the internal electric field is established.

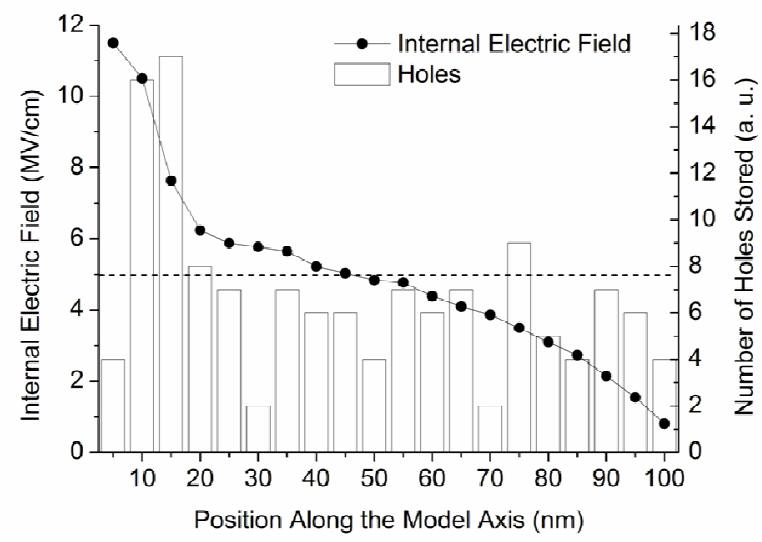

Fig. 2 - The hole and the internal electric field distribution along the model axis (direction perpendicular to the electrodes surface), for a polymer network where the chains are parallel to the electrodes. These results where obtained for an applied electric field of $5 \mathrm{MV} / \mathrm{cm}$ which is represented by the horizontal broken line.

In Fig. 2 we can see that, for a polymer layer with chain orientation parallel to the electrodes and for an applied electric field of $5 \mathrm{MV} / \mathrm{cm}$, hole distribution gives rise to a higher strength of the internal electric field near the cathode as compared to the anode. This asymmetric distribution of the internal electric field along the model axis results from the fact that this field is the sum of the electric field due to space charge distribution, the applied electric field and the field from electrode polarization. 


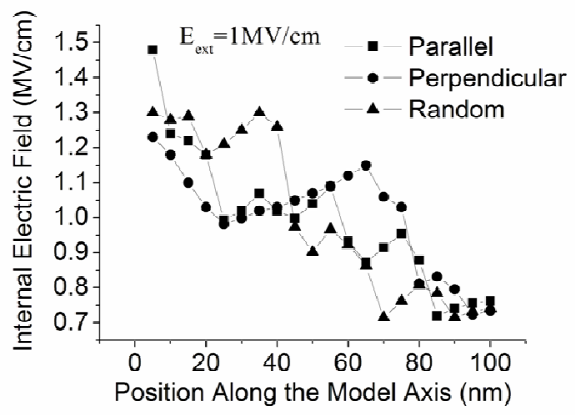

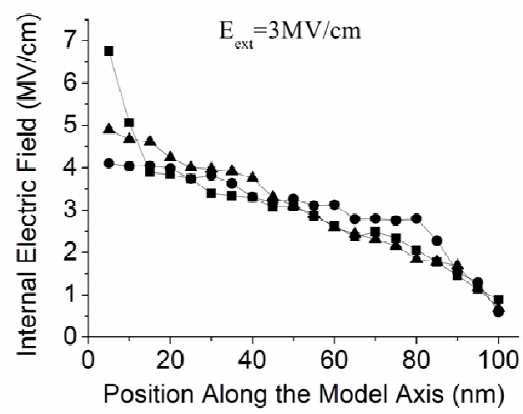

(b)

(a)

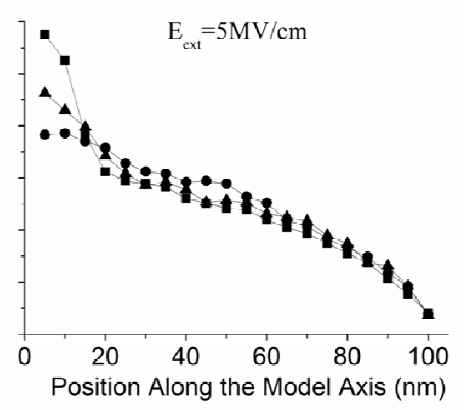

(c)

Fig. 3 - Internal electric field along the model axis for polymer layers with chain orientation relative to the electrode surface parallel (squares), perpendicular (circles) and random oriented (triangles) and three different strengths of the applied electric field ( $\left.\mathrm{E}_{\text {ext. }}\right)$.

Fig. 3 shows that hole distribution in the polymer network always leads to a gradient in the internal electric field along the model, regardless of the strength of the applied electric field, but the polymer morphology seems to influence the profile of the internal electric field especially near the region close to the cathode.

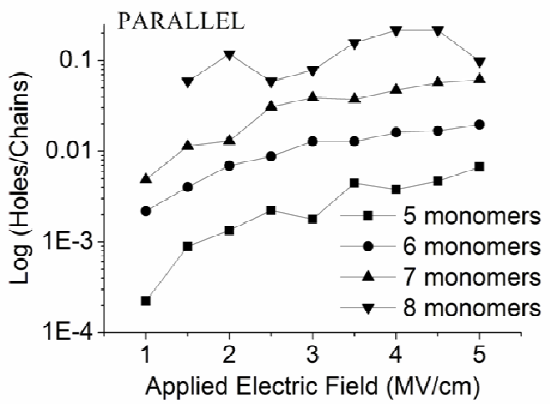

(a)

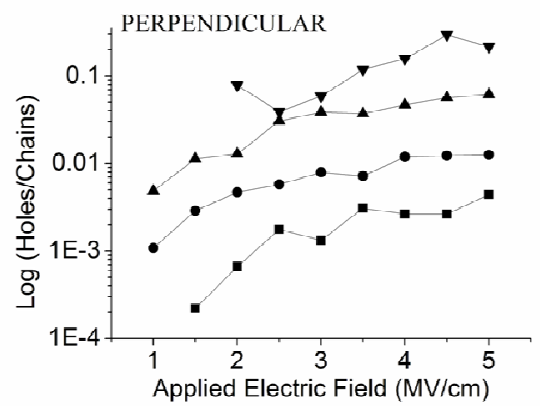

(b)

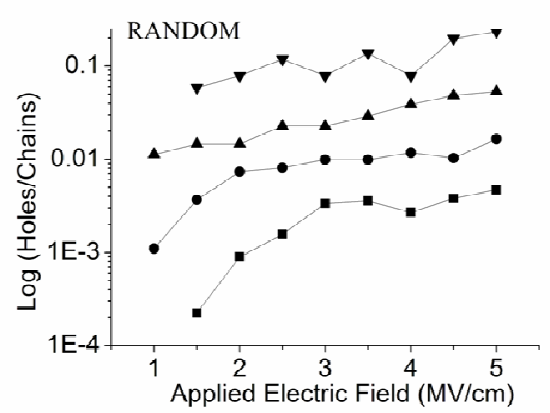

(c)

Fig. 4 - Number of holes stored per chain with a specific length, in logarithmic scale, as a function of the applied electric field, for the following chain alignments relative to the electrode surfaces: (a) parallel, (b) perpendicular and (c) random orientation. The lines are just a guide to the eye. 
The reason for this behaviour lies not only on the amount of holes stored but also on their distribution through the polymer layer. In all polymer layers used in our simulations, the number of chains with 5 monomer units is significantly higher than chains with other lengths, but the number of holes stored per chain with a specific length increases as the length of the chain increases. In Fig. 4 we can see that, for all three polymer chain orientations, chains with 8 monomers have the highest hole/chain ratio. This ratio increases with the strength of the applied electric field. The reason for this behaviour is related to the molecular properties of the polymer chains and the Gaussian distribution of lengths with a mean value of 5 monomers, which allow that holes stay stored for long in chain segments with 8 monomers. When a hole jumps between two molecular sites it needs to tunnel through a potential barrier that depends on the electric potential that results from space charge distribution and image charge due to electrode polarization, the applied electric potential and the difference between ionization potential of the polymer strands involved. These potential barriers will create wells and path ways that will trap holes preferentially in polymer strands with 8 monomers. Since there are few of these chains and they are randomly distributed through the length of each sample, the charge distribution is similar in all three polymer morphologies considered in this work.

\section{Conclusions}

In this paper we have showed that varying the orientation of polymer chains relative to the electrode surface can influence hole percolation in PPV layers, until an electron is injected. Polymer layers with chains parallel to the electrodes can store a higher number of holes and their distribution through the polymer layer gives rise to a higher internal electric field near the cathode. The reason for this behaviour lies on the fact the hole percolation in layers with this morphology is mainly driven by the hopping process between strands. Our results show that, independently of the polymer morphology, holes are preferentially stored in chain segments with 8 monomer units even though the polymer network has a very small number of these segments. Therefore, these longer polymer strands act as a kind of hole trap. The generic trends described here should be representative of other conducting polymeric materials.

\section{Acknowledgement}

This work is part of the research projects POCTI/CTM/41574/EEI/2005 and CONCREEQ/443/2001, approved by the Portuguese Foundation for Science and Technology (FCT) and POCTI and support by the European Community Fund FEDER. One of us (H.M.C.B.) is also indebted to FCT for financial support under PhD grant No SFRH/BD/22143/2005.

\section{References}

[1] Y. Shi, J. Liu and Y. Yang: J. Appl. Phys. 87/9 (2000) p. 4254.

[2] G. Teyssedre and C. Laurent: IEEE Transactions on Dielectrics and Electrical Insulation 12/5 (2005) p. 857.

[3] E.J. Samuelsen and J. Mardalen: Handbook of Organic Conductive Molecules and Polymers, (Wiley, Chichester 1997).

[4] A.M. Stoneham, M.M.D. Ramos, A.M. Almeida, H.M.G. Correia, R.M. Ribeiro, H. Ness and A.J. Fisher: J. Phys. Condens. Matter 14/42 (2002) p. 9877.

[5] M.M.D. Ramos and H.M.G. Correia: J. Phys. Condens. Matter 18/16 (2006) p. S429.

[6] M.M.D. Ramos and A.M. Stoneham: Comput. Mater. Sci. 17/2-4 (2000) p. 260. 